Ambiente \& Água - An Interdisciplinary Journal of Applied Science
ISSN 1980-993X - doi:10.4136/1980-993X
www.ambi-agua.net
E-mail: ambi.agua@gmail.com

\title{
Índices de qualidade da água e de estado trófico do rio Caiabi, MT
}

\author{
doi:10.4136/ambi-agua.1769 \\ Received: 02 Oct. 2015; Accepted: 30 Nov. 2015
Grasiane Andrietti $^{1}$; Rosane Freire ${ }^{2}$; Adriana Garcia do Amaral ${ }^{1}$; Frederico Terra de Almeida ${ }^{1}$; Milene Carvalho Bongiovani ${ }^{1}$; Roselene Maria Schneider ${ }^{1^{*}}$ \\ ${ }^{1}$ Universidade Federal de Mato Grosso (UFMT), Sinop, MT, Brasil \\ ${ }^{2}$ Universidade Estadual Paulista "Júlio de Mesquita Filho" (UNESP), Presidente Prudente, SP, Brasil \\ Departamento de Física, Química e Biologia \\ *Autor correspondente: e-mail: roselenems@yahoo.com.br, \\ grasiane_melo@yahoo.com.br, rofreire@gmail.com, adrianagamaral@gmail.com, fredterra@ufmt.br, \\ milene.bongiovani@gmail.com
}

\section{RESUMO}

Neste trabalho objetivou-se identificar a qualidade da água superficial do rio Caiabi, MT por meio do índice de qualidade de água (IQA) e índice de estado trófico (IET), bem como a avaliação das variações sazonais e espaciais, com a definição do delineamento do monitoramento mais adequado para o local de estudo. As coletas para o monitoramento da qualidade da água ocorreram em cinco pontos no rio Caiabi, entre julho de 2012 e junho de 2013. Foram quantificados os seguintes parâmetros de qualidade: $\mathrm{pH}$, temperatura, condutividade elétrica, oxigênio dissolvido, coliformes totais e termotolerantes, turbidez, nitrogênio Kjeldal, nitrito, nitrato, fósforo total, demanda bioquímica de oxigênio, série de sólidos e clorofila a. Os procedimentos de coleta e análises seguiram os definidos pelo Standard Methods for the Examination of Water and Wastewater. Os resultados dos índices IQA mostraram que as águas do rio Caiabi possuem boa qualidade. Os resultados de IET evidenciaram o baixo risco de eutrofização no rio Caiabi, com indicação de um ambiente lótico ultraoligotrófico. A análise de variância demonstrou que 10 dos 16 parâmetros de qualidade monitorados apresentaram diferenças de médias entre as estações de seca e chuva ou entre os pontos monitorados ou na interação entre estações e pontos. Esses resultados indicam que duas coletas anuais em dois pontos podem ser suficientes para descrever o comportamento da qualidade da água na bacia, desde que as condições de uso e ocupação do solo não sejam modificadas.

Palavras-chave: água superficial, bacia amazônica, recursos hídricos, uso e manejo do solo.

\section{Water quality index and eutrophication indices of Caiabi River, MT}

\section{ABSTRACT}

The objective of this study was to evaluate the water quality of the Caiabi River based upon the water quality index (WQI) and the trophic state index (TSI), considering seasonal and spatial variations, with the aim of determining the most appropriate monitoring design for this study site. Sampling for water quality monitoring was conducted at five points on the 
Caiabi River from July 2012 to June 2013. Quality parameters quantified were as follows: $\mathrm{pH}$, temperature, conductivity, dissolved oxygen, total and thermotolerant coliforms, turbidity, Kjeldahl nitrogen, nitrite, nitrate, total phosphorus, biochemical oxygen demand, series of solids, and chlorophyll a. Sampling procedures and analysis followed the methods recommended by the Standard Methods for the Examination of Water and Wastewater. The WQI results showed that the quality of the Caiabi River water is good. TSI results demonstrated the low risk of eutrophication in the Caiabi River, indicating an ultraoligotrophic lotic environment. Analysis of variance showed that 10 of the 16 monitored quality parameters presented differences of means between the dry and rainy seasons or among the monitored points or in the interaction between seasons and points. These results indicate that two annual sampling collections at two points may be sufficient to describe the water quality behavior in the basin, as long as the conditions of land use are stable.

Keywords: amazon basin, soil use and management, surface water, water resources.

\section{INTRODUÇÃO}

Pesquisas realizadas em bacias hidrográficas rurais têm demonstrado o potencial de degradação da qualidade das águas superficiais (Donadio et al., 2005; Chaves e Santos, 2009; Bateni et al., 2013; Rajankar et al., 2011). No Brasil, a região da Amazônia Legal sente as consequências do uso e ocupação do solo estabelecido aliado ao regime hídrico da região. Essas condições proporcionam um potencial teórico de degradação acentuado, considerando, principalmente, os eventos de intensa precipitação local (Souza et al., 2013). O transporte de sedimentos, o carreamento de nutrientes e matéria orgânica para o leito do rio traz agravos que podem se tornar irreversíveis, superando a capacidade de autodepuração do rio (Wittman et al., 2013).

Entre as bacias hidrográficas da região da Amazônia Legal, a do rio Caiabi chama a atenção por estar em uma região de grande importância social, econômica e ambiental. Localizado na região norte do estado de Mato Grosso, entre os biomas Amazônia e Cerrado (Marimon et al., 2006), é um curso d'água perene, de segunda ordem, possui $70 \mathrm{~km}$ de extensão e drena uma área de $499 \mathrm{~km}^{2}$, abrangendo os municípios de Sinop e Vera. O principal uso do solo dessa área é voltado para o plantio de soja e milho, culturas de extrema importância para a economia do estado de Mato Grosso, sendo a região norte um grande exportador de commodities agrícolas (Morales et al., 2013).

Quando a floresta é retirada dando lugar a sistemas de produção agrícola, ou pastagens, não apenas a paisagem é alterada, mas a qualidade da água da bacia hidrográfica também sofre modificações. Os usos múltiplos da água e as atividades antrópicas realizadas em uma bacia hidrográfica causa significativas alterações na qualidade dos recursos hídricos. Em prática, pouco se sabe sobre a qualidade das águas da bacia do rio Caiabi, o que representa que não se sabe qual é o real impacto do uso e ocupação do solo da bacia hidrográfica.

Em geral, decretos e resoluções sobre qualidade da água consistem em parâmetros de poluição das massas de água em monitoramento. Geralmente, são informações pouco expressivas para os cientistas, no entanto, significativas para os gestores e tomadores de decisão do setor de água e que querem saber sobre o estado de seus corpos hídricos (Nasirian, 2007). Nas últimas décadas, tem-se observado o crescente aumento de estudos quanto ao monitoramento da qualidade da água, como visto nos trabalhos realizados por Cunha et al. (2004), Prado e Moraes (2007), Pontes et al. (2012), Bucci e Oliveira (2014), em várias regiões do país. 
Desenvolvidos para integrar, interpretar e comunicar os dados obtidos no monitoramento ambiental, as análises estatísticas e os índices têm sido usados com sucesso para caracterizar o estado e as tendências da qualidade da água e transmitir essas informações para os grupos interessados. Não exigem grande número de parâmetros de qualidade da água para o desenvolvimento e a validação é necessária apenas para a concentração de um número limitado de parâmetros de qualidade da água.

A luz das problemáticas ambientais que envolvem a bacia hidrográfica do Rio Caiabi, com uso e ocupação predominantemente rural, o índice de qualidade de água (IQA) e o índice de estado trófico (IET) foram escolhidos nesse trabalho por representarem em um único número e a escala qualitativa correspondente, o estado da água de forma eficaz. Essa pesquisa também traz uma profunda avaliação das variações sazonais e espaciais da qualidade da água superficial do rio Caiabi, com a definição do delineamento experimental mais adequado para o local de estudo.

\section{MATERIAIS E MÉTODOS}

\section{1. Área de estudo}

A área de estudo foi a microbacia hidrográfica do rio Caiabi, localizada na região médio Norte do Estado de Mato Grosso, na área de abrangência da Amazônia Legal. O principal rio da microbacia é o Caiabi, com aproximadamente $51 \mathrm{~km}$ de extensão. Esta região apresenta clima tropical, de acordo com a classificação climática de Köppen-Geiger apresentando duas estações. Entre os meses de outubro a abril tem-se a estação de chuvas, sendo o restante do período caracterizado como seco, apresentando pouca ou nenhuma precipitação. A precipitação total anual é de aproximadamente $2.000 \mathrm{~mm}$ (Souza et al., 2013). O uso do solo da bacia é predominantemente de cultivo temporário, apresentando também áreas de pastagens.

Para as amostragens foram selecionados 5 locais de coleta ao longo canal principal do rio, considerando os aspectos de logística e acessibilidade ao local de coleta nos meses de seca e de chuva. Os locais foram denominados $\mathrm{P} 1, \mathrm{P} 2, \mathrm{P} 3, \mathrm{P} 4$ e $\mathrm{P} 5$, sendo o $\mathrm{P} 1$ próximo à nascente e o P5 próximo ao exutório da bacia (Figura 1).

Em relação aos locais escolhidos como pontos de monitoramento, o trecho definido como ponto 2 era o que apresentava maior área com influência de pastagens. Estradas rurais e carreadores foram identificados em todos os trechos que contribuíam para os pontos de coleta.

As campanhas de amostragem foram realizadas durante doze meses consecutivos, entre julho de 2012 e junho de 2013. A coleta da água era realizada no ponto central da seção transversal do rio à profundidade de $10 \mathrm{~cm}$, aproximadamente. Os parâmetros de qualidade investigados foram o oxigênio dissolvido (OD), turbidez (TB), pH, temperatura (T), coliformes totais (CT), coliformes termotolerantes (CTERM), sólidos totais (ST), sólidos dissolvidos (SD), sólidos suspensos (SS) demanda bioquímica de oxigênio (DBO), condutividade elétrica $(\mathrm{CE})$, nitrogênio total $(\mathrm{NT})$, nitrito $\left(\mathrm{NO}_{2}\right)$, nitrato $\left(\mathrm{NO}_{3}\right)$, fósforo total (PT) e clorofila (CL).

A concentração de oxigênio dissolvido (OD), a turbidez, a temperatura e o pH foram determinados in situ, por meio de sonda multiparâmetros Horiba. As amostras de água coletadas para a determinação dos outros parâmetros foram armazenadas em frascos de vidro ou polietileno e transportadas para a Universidade Federal de Mato Grosso campus Sinop em caixa de isopor contendo gelo. As coletas, preservações, transporte e procedimentos analíticos seguiram o descrito em APHA et al. (2005). 


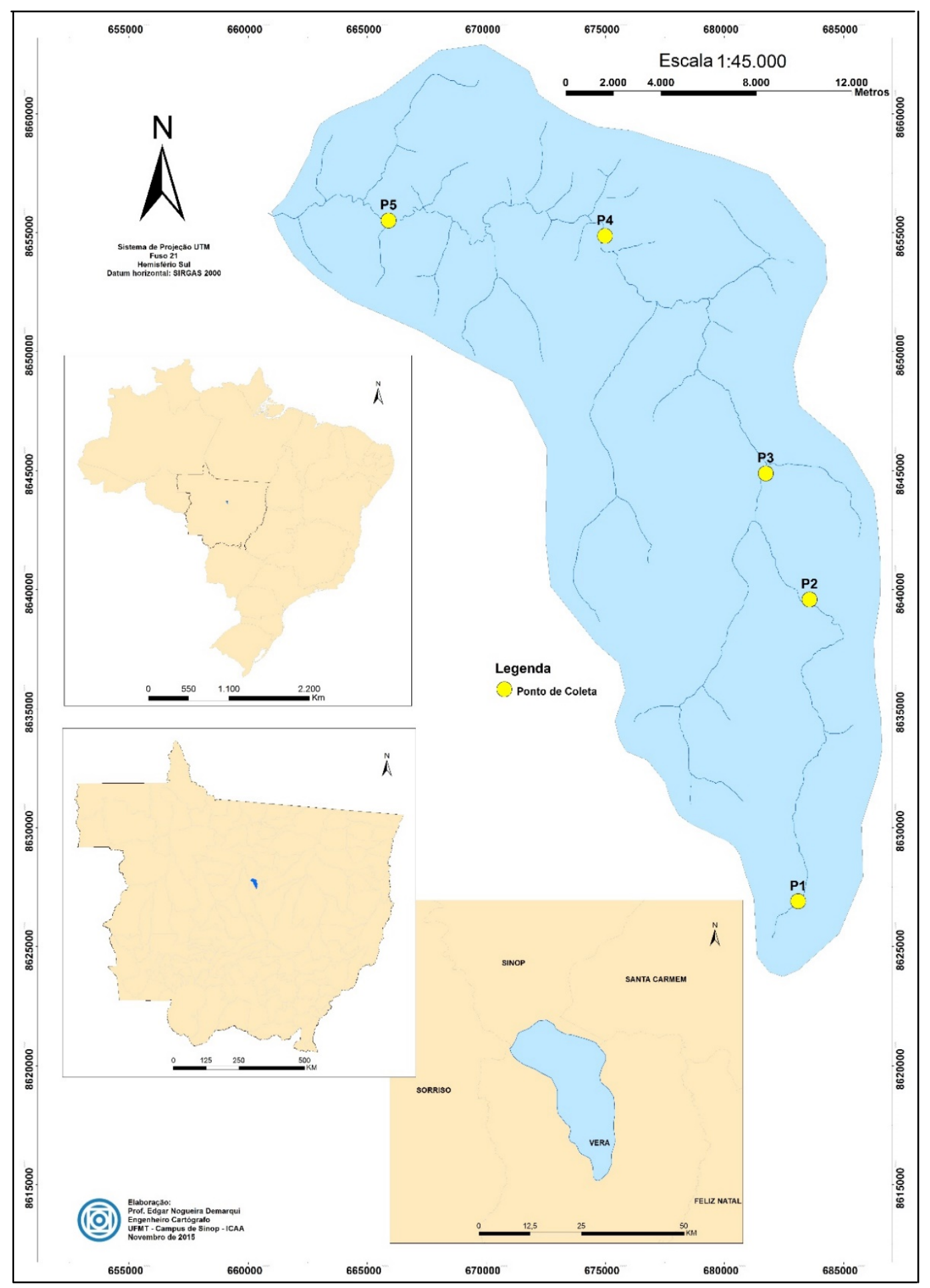

Figura 1. Localização regional da bacia hidrográfica do rio Caiabi no estado de Mato Grosso, com a ampliação da área da bacia e identificação dos pontos de amostragem.

Às amostras coletadas para análise de coliformes foram acondicionadas em frascos de Schott esterilizados. As amostras para análise de nutrientes (nitrogênio e fósforo) foram acidificadas logo após a coleta, com ácido sulfúrico. O método para as análises de coliformes totais e termotolerantes foi a de tubos múltiplos; o nitrogênio foi determinado pelo método 
micro-kjeldahl; o fósforo total pelo método do ácido ascórbico; o nitrito foi determinado pelo métdo colorimétrico; o nitrato pelo método de redução de cádmio; a demanda bioquímica de oxigênio pela incubação de amostras por cinco dias, a $20^{\circ} \mathrm{C}$; a condutividade elétrica foi medida em condutivímetro de bancada; para a determinação da clorofila a amostra foi filtrada em filtro de fibra de vidro, com posterior extração com acetona e determinação em espectrofotômetro; a determinação dos sólidos totais foi realizada por meio gravimétrico; os sólidos suspensos foram determinados pela filtração da amostra em filtro de fibra de vidro, secagem e pesagem; e os sólidos dissolvidos pela diferença entre sólidos totais e suspensos.

\subsection{Delineamento experimental e análise estatística}

Para verificar o efeito das fontes de variação (pontos de coleta e estações do ano) sobre os parâmetros de qualidade da água utilizou-se a análise de variância. Os testes foram realizados por meio do programa computacional $\mathrm{R}$ ( $R$ Development Core Team, 2011). O delineamento experimental adotado foi o Delineamento em Blocos ao Acaso, sendo que os tratamentos foram arranjados em esquema fatorial $5 \times 2$ : cinco pontos de coleta $\left(\mathrm{P}_{1}, \mathrm{P}_{2}, \mathrm{P}_{3}, \mathrm{P}_{4} \mathrm{e}\right.$ $\mathrm{P}_{5}$ ) e duas estações (seca: abril a setembro e chuvosa: outubro a março), com seis repetições. Nos meses de seca as precipitações foram muito pequenas $(<10 \mathrm{~mm}$ em abril e setembro) e nulas em maio, junho, julho e agosto. Após a análise de variância (ANOVA), os parâmetros que apresentaram diferença estatisticamente significativa $(\mathrm{p}<0,05)$ foram submetidos ao teste de Tukey para comparação de médias e o desdobramento da análise fatorial.

\section{3. Índice de qualidade da água e índice de estado trófico}

O Índice de Qualidade da Água é uma indicação aproximada do estado de qualidade do corpo hídrico. Para a determinação do IQA utilizou-se a Equação 1 (CETESB, 2008).

$$
I Q A=\prod_{i=1}^{9} Q_{i}^{w}
$$

em que:

$\mathrm{Q}_{\mathrm{i}}$ é o valor de qualidade do i-ésimo parâmetro, um número entre 0 e 100, obtido da respectiva curva média de variação de qualidade, em função de sua concentração ou medida (ANA, 2015) e;

wi é o peso correspondente ao i-ésimo parâmetro fixado em função da sua importância para a conformação global da qualidade, isto é, um número entre 0 e 1.

Cada um dos parâmetros que constitui o IQA possui um determinado peso, relativo à medida da sua contribuição para a qualidade da água. Os valores utilizados foram aqueles apresentados em ANA (2015).

A qualidade da água será em função do valor de IQA obtido, podendo ser de péssima (IQA $<25)$, ruim $(26<$ IQA $<50)$, regular $(51<$ IQA $<70)$, boa $(71<$ IQA $<90)$ ou ótima qualidade $(91<\mathrm{IQA} \leq 100)(\mathrm{ANA}, 2015)$.

O Índice de Estado Trófico (IET) auxilia na visualização geral grau de trofia (nutrição) e seu efeito na qualidade da água. O IET foi determinado com os resultados de clorofila $a$ (CL) e fósforo total (PT), seguindo o método descrito por Alves et al. (2012). As Equações 2, 3 e 4 foram utilizadas para a determinação do IET. 


$$
\begin{aligned}
& \operatorname{IET}(P T)=10 \cdot\left\{6-\left[\frac{0,42-0,36 \cdot \ln (P T)}{\ln (2)}\right]\right\}-20 \\
& \operatorname{IET}(C L)=10 \cdot\left\{6-\left[\frac{-0,7-0,6 \cdot \ln (C L)}{\ln (2)}\right]\right\}-20 \\
& \operatorname{IET}=\frac{\operatorname{IET}(P T)+\operatorname{IET}(C L)}{2}
\end{aligned}
$$

em que:

IET (PT) é o índice de estado trófico determinado para o fósforo;

IET (CL) é o índice de estado trófico determinado para a clorofila;

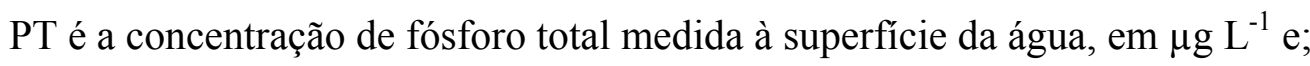

CL é a concentração de clorofila $a$ medida à superfície da água, em $\mu \mathrm{g} \mathrm{L^{-1 }}$.

A classificação do ambiente aquático quanto ao IET é dada em seis graus de trofia. Para rios tem-se: ultraoligotrófico (IET $\leq 47)$, oligotrófico $(47<$ IET $\leq 52)$, mesotrófico $(52<$ IET $\leq 59)$, eutrófico $(59<$ IET $\leq 63)$, supereutrófico $(63<$ IET $\leq 67)$ e hipereutrófico $($ IET $>67)$ (ANA, 2015).

\section{RESULTADOS E DISCUSSÃO}

\subsection{Variação sazonal e temporal dos parâmetros indicativos de qualidade hídrica}

A análise de variância mostrou que, dos parâmetros indicativos de qualidade analisados, sete apresentaram diferenças médias de concentração entre as estações seca e chuvosa $(p<0,05)$. As diferenças médias entre parâmetros foram identificadas nos parâmetros coliformes totais, DBO, nitrito, turbidez, temperatura, sólidos dissolvidos e sólidos totais (Tabela 1).

Tabela 1. Valores médios dos parâmetros indicativos de qualidade monitorados em função da estação.

\begin{tabular}{lccc}
\hline \multirow{2}{*}{ Parâmetro } & \multicolumn{2}{c}{$\begin{array}{c}\text { Valores médios nas } \\
\text { estações }\end{array}$} & $\begin{array}{c}\text { Valores de } \\
\text { referência* }\end{array}$ \\
\cline { 2 - 3 } & Chuva & Seca & \\
\cline { 1 - 3 } $\mathrm{CT}\left(\mathrm{NMP} 100 \mathrm{~mL}^{-1}\right)$ & 33,0 & 9,0 & -- \\
$\mathrm{DBO}\left(\mathrm{mg} \mathrm{L}^{-1}\right)$ & 1,2 & 0,5 & $<3$ \\
$\mathrm{NO}_{2}\left(\mathrm{mg} \mathrm{L}^{-1}\right)$ & 2,6 & 2,1 & $<1^{1}$ \\
$\mathrm{~TB}(\mathrm{NTU})$ & 11,36 & 6,26 & $<40$ \\
$\mathrm{~T}\left({ }^{\circ} \mathrm{C}\right)$ & 25,53 & 22,48 & $40 \pm 3{ }^{\circ} \mathrm{C}$ \\
$\mathrm{SD}\left(\mathrm{mg} \mathrm{L}^{-1}\right)$ & 68,0 & 29,0 & $<500$ \\
$\mathrm{ST}\left(\mathrm{mg} \mathrm{L}^{-1}\right)$ & 86,0 & 35,0 & -- \\
\hline
\end{tabular}

Nota: *Resolução CONAMA n $357 / 2005$.

Os poluentes $\mathrm{CT}, \mathrm{DBO}, \mathrm{NO}_{2}, \mathrm{~TB}, \mathrm{SD}$ e $\mathrm{ST}$ que apresentaram diferença significativa entre as estações revelaram a tendência de valores mais elevados durante o período de chuvas. 
Este comportamento é reflexo, principalmente, da ação do escoamento superficial das áreas agrícolas e de pastagens pela ocorrência de eventos de precipitação, em contraste com a estação seca.

Maiores concentrações de poluentes em função da sazonalidade estão descritas em literatura. Para os parâmetros coliformes totais (De Azevedo Lopes e Magalhães Jr., 2010; Chaves e Santos, 2009), DBO (Donadio et al., 2005), nitrito (Berka et al., 2001), turbidez, sólidos totais (Ríos-Villamizar et al., 2011; De Azevedo Lopes e Magalhães Jr., 2010), os autores reportam maiores concentrações nos períodos de chuva. De acordo com Grossi (2006), as estradas vicinais e carreadores servem de caminho para o escoamento superficial, ocasionando os processos erosivos, que se intensificam na estação chuvosa devido ao aumento na quantidade e na velocidade do fluxo.

Durante a estação seca os rios são abastecidos preponderantemente pelo escoamento subsuperficial, reduzindo a quantidade de sólidos e, consequentemente, as moléculas poluentes que adentram os recursos hídricos, aderidas às partículas coloidais. Apesar de ter sido identificada diferenças de concentrações entre estações, tem-se que os valores de referência para rios de classe 1 não foram ultrapassados.

A não observação de diferenças médias entre pontos, e somente entre estações, é um resultado bastante importante, pois indica que o acompanhamento do comportamento da qualidade da água não necessita de monitoramento constante (mensal). Duas coletas anuais em um único ponto são suficientes para demonstrar a qualidade da água em termos gerais, salvo situações de utilização pontual e específica da água.

Essa condição é de extrema importância, uma vez que a manutenção de um programa de monitoramento é dispendiosa. Se o monitoramento tem custos reduzidos, a possibilidade de manutenção da ação de monitoramento é maior.

Assim, para fins de acompanhamento do comportamento da qualidade da água e do estado trófico, a seção do rio próxima à confluência com o rio Teles Pires (P5) poderia ser uma estação constante de acompanhamento da qualidade da água, uma vez que este trecho apresenta uma seção de medição de vazão em operação desde o ano de 2010.

Em relação aos pontos de amostragem, os parâmetros indicativos de qualidade que apresentaram diferença significativa entre os trechos monitorados foram DBO e fósforo total (Tabela 2).

Tabela 2. Valores médios dos parâmetros indicativos de qualidade monitorados em função do local de amostragem.

\begin{tabular}{ccccc}
\hline \multirow{2}{*}{ Ponto } & \multicolumn{4}{c}{ Valores Médio } \\
\cline { 2 - 5 } & \multirow{2}{*}{$\mathrm{DBO}\left(\mathrm{mg} \mathrm{L}^{-1}\right)^{*}$} & $\mathrm{PT}\left(\mathrm{mg} \mathrm{L}^{-1}\right)^{*}$ & \multicolumn{2}{c}{ Sólidos suspensos $\left(\mathrm{mg} \mathrm{L}^{-1}\right)^{* *}$} \\
\hline $\mathrm{P}_{1}$ & $1,1 \mathrm{ab}$ & $0,09 \mathrm{ab}$ & $6,3 \mathrm{bA}$ & $35,0 \mathrm{aA}$ \\
$\mathrm{P}_{2}$ & $1,4 \mathrm{a}$ & $0,24 \mathrm{a}$ & $5,0 \mathrm{bA}$ & $21,7 \mathrm{aAB}$ \\
$\mathrm{P}_{3}$ & $0,8 \mathrm{ab}$ & $0,09 \mathrm{ab}$ & $3,8 \mathrm{aA}$ & $6,7 \mathrm{aB}$ \\
$\mathrm{P}_{4}$ & $0,8 \mathrm{ab}$ & $0,14 \mathrm{ab}$ & $7,5 \mathrm{aA}$ & $14,2 \mathrm{aB}$ \\
$\mathrm{P}_{5}$ & $0,6 \mathrm{~b}$ & $0,06 \mathrm{~b}$ & $6,3 \mathrm{aA}$ & $11,7 \mathrm{aB}$ \\
\hline
\end{tabular}

Nota: * Médias seguidas de mesma letra minúscula na coluna não apresentam diferença significativa. ${ }^{* *}$ Médias seguidas por letra minúscula na linha e maiúscula na coluna não diferem entre si a $5 \%$ de significância.

Os valores médios de concentração de $\mathrm{DBO}$ e fósforo total mostraram que os pontos $\mathrm{P}_{1}$, $\mathrm{P}_{3}$ e $\mathrm{P}_{4}$ apresentam comportamento semelhante entre si, enquanto os pontos $\mathrm{P}_{2}$ e $\mathrm{P}_{5}$ diferem completamente entre si e dos demais. $\mathrm{O}_{2}$ está situado logo abaixo de uma região com 
intensa criação animal, e à jusante do município de Vera (MT), fator que pode explicar o incremento de matéria orgânica e fósforo total no leito fluvial. Verificou-se que para o parâmetro fósforo, a qualidade da água estava em desacordo com o estipulado pela Resolução CONAMA n ${ }^{\circ} 357$, de 2005, que prevê concentração máxima de $0,1 \mathrm{mg} \mathrm{L}^{-1}$.

Toledo e Nicolella (2002) também constataram diferenças significativas entre pontos de água superficial monitorados. Zanini et al. (2010) observaram diferença significativa na análise de DBO entre os pontos amostrados e na interação entre pontos e estações.

A observação de diferenças médias desses parâmetros em relação aos pontos demonstra que, além de se monitorar o ponto 5, é preciso monitorar trecho a montante na bacia como, por exemplo, o_P2.

Observou-se que o efeito da interação do ponto de amostragem com a estação como fonte de variação para os parâmetros de qualidade somente ocorreu para o parâmetro sólidos suspensos. Comparando os valores médios de sólidos suspensos verificou-se maiores concentrações para os pontos 1 e 2 no período de chuva, enquanto que para os outros pontos esta diferença não ocorreu (Tabela 2).

A ocorrência das elevadas concentrações é dada em consequência das alterações antrópicas no local durante o período de monitoramento. $\mathrm{O}$ trecho representado pelo ponto 1 sofreu algumas intervenções ao longo do período de monitoramento. Porém, as alterações não se refletiram nos outros trechos provavelmente devido às características do canal, com possíveis pontos de menor velocidade que favoreceram a sedimentações dos sólidos. A produção de sedimentos está intimamente relacionada com a descarga líquida e com a combinação de fatores morfométricos da bacia (Antoneli e Thomaz, 2007). Segundo Chapman (1996), a concentração dos sólidos suspensos varia dramaticamente com as mudanças na descarga líquida da bacia.

No período de seca observou-se que a concentração de sólidos suspensos mantém-se constante ao longo de todo o canal. Ou seja, sem chuvas não se tem escoamento superficial e como a vazão mantém-se com pouca alteração, o arraste de sólidos nas águas do rio apresenta-se constante.

Os resultados do monitoramento para os outros elementos monitorados, clorofila a, coliformes termotolerantes, oxigênio dissolvido, condutividade elétrica e $\mathrm{pH}$, não apresentaram valores médios fora dos limites estabelecidos pela Resolução Conama ${ }^{\circ} 357$ de 2005 (Tabela 3), exceto para o $\mathrm{pH}$.

Tabela 3. Valores médios dos poluentes monitorados na bacia hidrográfica do rio Caiabi.

\begin{tabular}{lcccccc}
\hline Parâmetro & $\begin{array}{c}\text { Conc. } \\
\text { Média }\end{array}$ & DP & $\begin{array}{c}\text { Conc. } \\
\text { Mín. }\end{array}$ & $\begin{array}{c}\text { Conc. } \\
\text { Máx. }\end{array}$ & CV (\%) & $\begin{array}{c}\text { Valores de } \\
\text { Referência* }\end{array}$ \\
\hline $\mathrm{CL}\left(\mathrm{mg} \mathrm{L}^{-1}\right)$ & 0,43 & 0,49 & 0,00 & 2,80 & 0,68 & $<10,0$ \\
$\left.\mathrm{CTERM} \mathrm{(NMP} 100 \mathrm{~mL}^{-1}\right)$ & 4,02 & 2,24 & 3,00 & 23,00 & 1,56 & $<200,0$ \\
$\mathrm{NO}_{3}\left(\mathrm{mg} \mathrm{L}^{-1}\right)$ & 3,15 & 1,73 & 0,24 & 8,87 & 0,55 & $<10,0$ \\
$\mathrm{OD}\left(\mathrm{mg} \mathrm{L}^{-1}\right)$ & 6,61 & 1,33 & 4,30 & 10,50 & 0,21 & $>6,0$ \\
$\mathrm{CE}\left(\mu{\left.\mathrm{S} . \mathrm{cm}^{-1}\right)}_{\mathrm{pH}}^{3,31}\right.$ & 3,61 & 1,00 & 19,00 & 1,09 & -- \\
\hline
\end{tabular}

Nota. DP: Desvio-padrão; CV: Coeficiente de variação. * Resolução CONAMA 357 (2005) Segundo Frota Júnior et al. (2007), a condutividade elétrica é um parâmetro afetado por efeito acumulativo de sais totais. Portanto, poder-se-ia esperar resultados superiores no sentido nascente-foz, o que não foi observado nas águas do rio Caiabi. 
Apesar do valor médio do $\mathrm{pH}$ estar abaixo do que é indicado pela legislação, sabe-se que não é o uso e ocupação do solo que faz com que o pH seja baixo, e sim uma condição natural da bacia. Os solos da região são solos antigos e basicamente todas as bases do solo já foram lixiviadas (EMBRAPA, 2006).

Em relação aos parâmetros descritos na Tabela 3, a não observação de diferenças indica que o acompanhamento desses em um único ponto, com uma coleta anual é suficiente para demonstrar o comportamento deles.

\section{2. Índice de qualidade da água e índice estado trófico}

Apesar de não ter sido identificada diferença significativa entre pontos e estações, para fins de indicação da classe em termos de IQA e IET, valores médios dos pontos em cada uma das estações foram calculados. Estes valores indicaram que os trechos do rio Caiabi apresentaram boa qualidade da água, com baixo potencial de desenvolvimento de processo de eutrofização, conforme resultados apresentados na Tabela 4.

Tabela 4. Classificação da água do rio Caiabi em função do IET e IQA.

\begin{tabular}{cccccc}
\hline Ponto & Estação & IET & Classificação & IQA & Classificação \\
\hline \multirow{2}{*}{ P1 } & Seca & 26 & Ultraoligotrófico & 76 & Boa \\
& Chuva & 22 & Ultraoligotrófico & 74 & Boa \\
P2 & Seca & 20 & Ultraoligotrófico & 75 & Boa \\
& Chuva & 29 & Ultraoligotrófico & 74 & Boa \\
P3 & Seca & 29 & Ultraoligotrófico & 76 & Boa \\
& Chuva & 21 & Ultraoligotrófico & 74 & Boa \\
P4 & Seca & 17 & Ultraoligotrófico & 81 & Boa \\
& Chuva & 10 & Ultraoligotrófico & 76 & Boa \\
& Seca & 19 & Ultraoligotrófico & 87 & Boa \\
& Chuva & 25 & Ultraoligotrófico & 78 & Boa \\
\hline
\end{tabular}

Analisando a tendência temporal do IQA em 29 pontos de monitoramento na sub-bacia do rio das Velhas, Trindade (2013) constatou a influência da sazonalidade em apenas 3 pontos (10,34\%). Zanini et al. (2010) também não verificaram diferença significativa nos resultados médios de IQA entre as estações, entretanto, registrou diferenças entre os pontos analisados.

Inúmeros fatores possuem a propriedade de atenuar os efeitos negativos do uso do solo na qualidade da água. A presença de mata ciliar, segundo Donadio et al. (2005), auxilia na proteção dos recursos hídricos. O Caiabi possui mata ciliar ao longo de toda a sua extensão, o que pode ter contribuído com os altos resultados de IQA obervados. Outro fator que pode influenciar a qualidade da água é a cobertura vegetal. De acordo com Vanzela et al. (2010), as áreas de matas são áreas mais cobertas e estáveis, com maior capacidade de infiltração e armazenamento de água no solo, reduzindo o escoamento superficial.

É também importante ressaltar que com o início das chuvas há também o plantio da soja que ao nascer e se desenvolver protege o solo (cobertura) e que o manejo de solo (terraceamento) contribuem para a redução do carreamento de solo e outros elementos ligados a este.

O estudo de caso de Wu et al. (2013) confirmou a hipótese de que a reabilitação de águas poluídas com base no replantio de vegetação pode aliviar o aumento da poluição e possibilitar a reabilitação do ecossistema aquático degradado. 
Avaliando a ocupação no solo, fragmentação da paisagem e a qualidade da água da bacia do córrego Ponte de Terra (DF) entre os anos de 1992 e 2005, Chaves e Santos (2009), reportaram valores de IQA variando na faixa 71 - 81 no período de chuvas e 75 - 82 na estação seca. Os mesmos autores constataram um IQA ligeiramente superior no período de seca. Toledo e Nicolella (2002) observaram uma sutil deterioração na qualidade da água durante o período de chuvas, concluindo que os principais indicadores que contribuíram para os menores valores de IQA foram o OD, PT, amônia e CE. Magalhães Jr. et al. (2008) encontraram no ribeirão de Carrancas resultados de IQA inferiores aos encontrados no rio Caiabi, variando entre 37 e 64 . Os autores atribuíram a baixa qualidade da água à influência da atividade pecuária na região.

Em termos gerais, verificou-se, pelos valores de IET, que o nível trófico do rio se encontra pouco desenvolvido, pois os valores são baixos. De acordo com os resultados, as amostras coletadas apresentaram um índice ultraoligotrófico, sendo os valores de IET $<47$.

Affonso et al. (2011) encontraram valores de IET variando entre 32,5 e 75,5 na bacia hidrográfica do rio Taperoá, superiores aos valores encontrados no rio Caiabi. Cunha et al. (2008) avaliando a densidade fitoplanctônica e o estado trófico dos rios Canha e PariqueraAçu na bacia hidrográfica do rio Ribeira de Iguape (SP) concluíram que o IET aplicado a ambientes lóticos contribui para a superestimação dos resultados. Os autores afirmam que as formas fosfatadas que integram o cálculo podem contribuir para um incremento indevido do resultado, pois ocorre um aumento incompatível com a real resposta biológica associada, representada pelo crescimento fitoplanctônico.

O nível de estado trófico do rio Caiabi se encontra muito abaixo de uma faixa de risco e comprometimento em curto prazo. De acordo com Cunha et al. (2008), a eutrofização em ambientes lóticos seria incomum, devido as características próprias desse sistema, tais como o turbilhonamento, baixo tempo de residência e transporte contínuo da comunidade fitoplanctônica a jusante.

Os resultados da análise de variância para os índices de qualidade da água e de estado trófico demonstraram que não houve diferenças significativas dos valores entre os pontos, estações e nem na interação do conjunto. Apesar de ter-se identificado que concentrações dos elementos monitorados diferiram entre si em relação às estações e aos pontos, o efeito dessas diferenças nos índices obtidos foi inibido.

Quando índices são obtidos é importante considerar o efeito eclipse nos resultados. De acordo com Silva e Jardim (2006), o efeito eclipse advém do processo de agregar inúmeras variáveis ambientais em um único número, podendo atenuar o impacto negativo de uma das variáveis frente ao comportamento estável das demais.

A não observação de diferenças é um resultado bastante importante, pois indica que acompanhamento do comportamento da qualidade da água não necessita de monitoramento constante. Considerando apenas os resultados dos índices, uma única coleta anual em um único ponto é suficiente para demonstrar a qualidade da água em termos gerais, salvo situações de utilização pontual e específica da água.

Reitera-se que essa condição é importante, uma vez que a manutenção de um programa de monitoramento é dispendiosa. Se o monitoramento tem custos reduzidos, a possibilidade de manutenção da ação de monitoramento é maior.

Assim, para fins de acompanhamento do comportamento da qualidade da água e do estado trófico, a seção do rio próxima à confluência com o rio Teles Pires (P5) poderia ser uma estação constante de acompanhamento do índice de qualidade da água, uma vez que este trecho apresenta uma seção de medição de vazão em operação desde o ano de 2010.

Zhang et al. (2013) verificaram que a similaridade e a diferença temporal e espacial dos parâmetros podem permitir a otimização da frequência de monitoramento, o número de locais amostrados, de parâmetros monitorados, e, portanto, o custo associado. Assim, enquanto a 
bacia do rio Caiabi mantiver as mesmas atividades e mesmas práticas de uso e ocupação do solo, uma coleta anual no canal principal da bacia é suficiente para se identificar a qualidade da água em termos de IQA e IET.

\section{CONCLUSÕES}

Os resultados dos índices IQA apontam que as águas do rio Caiabi possuem qualidade boa. Os resultados podem ser atribuídos à presença de vegetação nativa, à conservação da mata ciliar e ao efeito de diluição motivado pelo acréscimo de tributários ao longo do trecho do rio.

Os resultados de IET evidenciam o baixo risco de eutrofização no rio Caiabi, uma vez o IET não excedeu o limite de 47. O ambiente pode ser classificado como ultraoligotrófico.

A análise de variância dos parâmetros monitorados demonstrou que o acompanhamento da qualidade da água pode ser realizado considerando-se apenas duas coletas anuais, uma no período de seca e outra no período de chuva, em dois pontos, sendo essas suficientes para descrever o comportamento da qualidade da água na bacia da maior parte dos parâmetros, desde que as condições de uso e ocupação do solo da bacia não sejam modificadas.

\section{AGRADECIMENTOS}

Ao Conselho Nacional de Desenvolvimento Científico e Tecnológico e à Coordenação de Aperfeiçoamento de Pessoal de Nível Superior pelo apoio financeiro.

\section{REFERÊNCIAS}

AFFONSO, A. G.; BARBOSA, C.; NOVO, E. M. L. M. Water quality changes in floodplain lakes due to the Amazon River flood pulse: Lago Grande de Curuaí (Pará). Brazilian Journal of Biology, v. 71, p. 601-610, 2011. http://dx.doi.org/10.1590/S151969842011000400004

AGÊNCIA NACIONAL DE ÁGUAS - ANA (Brazil). Portal da qualidade das águas. Indicadores de qualidade. Índice de qualidade das águas (IQA). 2015. Disponível em: http://portalpnqa.ana.gov.br/indicadores-indice-aguas.aspx. Acesso em: 15 abr. 2015.

ALVES, I. C. C.; El-ROBRINI, M.; SANTOS, M. L. S.; MONTEIRO; S. M.; BARBOSA, L. P. F.; GUIMARÃES, J. T. F. Qualidade das águas superficiais e avaliação do estado trófico do Rio Arari (Ilha de Marajó, norte do Brasil). Acta Amazônica, v. 42, n. 1, p. 115-124, 2012. http://dx.doi.org/10.1590/S0044-59672012000100014

AMERICAN PUBLIC HEALTH ASSOCIATION - APHA; AMERICAN WATER WORKS ASSOCIATION - AWWA; WATER ENVIRONMENT FEDERATION - WEF. Standard Methods for the Examination of Water and Wastewater. $14^{\text {th }} \mathrm{ed}$. Washington, D.C, 2005.

ANTONELLI. V.; THOMAZ, E. L. Caracterização do meio físico da bacia do arroio Boa Vista - Guamiranga, PR. Caminhos de Geografia, v. 8, p. 46-58, 2007.

BATENI, F.; FAKHERAN, S.; SOFFIANIAN, A. Assessment of land cover changes \& water quality changes in the Zayadhroud river basin between 1997-2008. Environmental Monitoring Assessment, v. 185, p. 10511-10519, 2013.

http://dx.doi.org/10.1007/s10661-013-3348-3 
BERKA, C.; SCHREIER, H.; HALL, K. Linking water quality with agricultural intensification in a rural watershed. Water, Air and Soil Pollution, v. 127, p. 389-401, 2001. http://dx.doi.org/10.1023/A:1005233005364

BUCCI, M. H. S.; OLIVEIRA, L. F. C. Índices de qualidade da água e de estado trófico na represa Dr. João Penido (Juiz de Fora, MG). Revista Ambiente \& Água, v. 9, n. 1, p. 130-148, 2014. http://dx.doi.org/10.4136/ambi-agua.1290

CHAPMAN, D. Water Quality Assessments: a guide to use of biota, sediments and water in environmental monitoring. $2^{\text {nd }}$ Edition. London: UNESCO/WHO/UNEP, 1996. p. 651.

CHAVES, H. M. L.; SANTOS, L. B. Ocupação do solo, fragmentação da paisagem e qualidade da água em uma pequena bacia hidrográfica. Revista Brasileira de Engenharia Agrícola e Ambiental, v. 13, p. 922-930, 2009.

http://dx.doi.org/10.1590/S1415-43662009000700015

COMPANHIA AMBIENTAL DO ESTADO DE SÃO PAULO - CETESB. Índices de qualidade das águas. 2008. Disponível em: http://www.cetesb.sp.gov.br/userfiles/ file/agua/aguas-superficiais/aguas-interiores/documentos/indices/01.pdf. Acesso em: 17 ago. 2014.

CONSELHO NACIONAL DE MEIO AMBIENTE - CONAMA. Resolução no 357, de 17 de março de 2005. Dispõe sobre a classificação dos corpos de água e diretrizes ambientais para o seu enquadramento, bem como estabelece as condições e padrões de lançamento de efluentes, e dá outras providências. Alterado pela Resolução CONAMA 397/2008. Disponível em: http://www.mma.gov.br/conama. Acesso em: 04 set. 2015.

CUNHA, D. G. F.; FALCO, P. B.; CALIJURI, M. C. Densidade fitoplanctônica e estado trófico dos rios Canha e Pariquera-Açu, bacia hidrográfica do rio Ribeira de Iguape, SP, Brasil. Revista Ambiente \& Água, v. 3, n. 2, 2008. http://dx.doi.org/10.4136/ambiagua. 55

CUNHA, A. C.; CUNHA, H. F. A.; BRASIL JUNIOR, A. C. P.; DANIEL, L. A. SCHULZ, H. E. Qualidade microbiológica da água em rios de áreas urbanas e periurbanas no baixo amazonas: o caso do Amapá. Engenharia Sanitária e Ambiental, v. 9, n. 4, p. 322-328, 2004. http://dx.doi.org/10.1590/S1413-41522004000400009

DE AZEVEDO LOPES, F. W.; MAGALHÃES JR., A. P. M. Influência das condições naturais de $\mathrm{pH}$ sobre o índice de qualidade das águas (IQA) na bacia do Ribeirão de Carrancas. Geografias, v. 6, n. 2, p. 134-147, 2010.

DONADIO, N. M. M.; GALBIATTI, J. A.; PAULA, R. C. Qualidade da água de nascentes com diferentes usos do solo na bacia hidrográfica do córrego Rico, São Paulo, Brasil. Engenharia Agrícola, v. 25, p. 115-125, 2005. http://dx.doi.org/10.1590/S010069162005000100013

EMPRESA BRASILEIRA DE PESQUISA AGROPECUÁRIA - EMBRAPA. Embrapa Arroz e Feijão. Cultivo do arroz de terras altas no estado de Mato Grosso. Sistemas de Produção, n. 7. 2006. Disponível em: https://sistemasdeproducao.cnptia.embrapa.br/ FontesHTML/Arroz/ArrozTerrasAltas/index.htm. Acesso em: 29 out. 2015.

FROTA JÚNIOR, J. I.; ANDRADE, E. M.; MEIRELES, A. C. M.; BEZERRA, A. M. E.; SOUZA, B. F. S. Influência antrópica na adição de sais no trecho perenizado da bacia hidrográfica do Curu, Ceará. Revista Ciência Agronômica, v. 38, p. 142-148, 2007. 
GROSSI, C. H. Diagnóstico e monitoramento ambiental da microbacia hidrográfica do rio Queima-Pé (MT). 2006. 122f. Tese (Doutorado em Ciências Agronômicas) Universidade Estadual Paulista Júlio De Mesquita Filho, Botucatu, 2006.

MAGAlHÃes JR., A. P.; DE AZEVEDO LOPES, F. W.; PEREIRA, J. A. A. P. Avaliação da qualidade das águas e condições de balneabilidade na bacia do ribeirão de CarrancasMG. Revista Brasileira de Recursos Hídricos, v. 13, p. 111-120, 2008.

MARIMON, B. S.; LIMA, E. S.; DUARTE, T. G.; CHIEGERATTO, L. C.; RATTER, J. A. Observations on the vegetation of Northeastern Mato Grosso, Brazil: an analysis of the Cerrado-Amazonian forest ecotone. Edinburgh Journal of Botany, v. 63, p. 323-341, 2006. http://dx.doi.org/10.1017/S0960428606000576

MORAlES, P. R. G. D.; D'AGOSTO, M. A.; SOUZA, C. D. R. Otimização de rede intermodal para o transporte de soja do norte do Mato Grosso ao porto de Santarém. Journal of Transport Literature, v. 7, n. 2, p. 29-51, 2013.

NASIRIAN, M. A new water quality index for environmental contamination contributed by mineral processing: a case study of Amang (Tin Tailing) processing activity. Journal of Applied Science, v. 7, n. 20, p. 2977-2987, 2007.

PONTES, P. P.; MARQUES, A. R.; MARQUES, G. F. Efeito do uso e ocupação do solo na qualidade da água na micro-bacia do Córrego Banguelo - Contagem. Revista Ambiente \& Água, v. 7, n. 3, p. 598-613, 2012. http://dx.doi.org/10.4136/ambiagua.962

PRADO, R. B.; MORAES NOVO, E. M. L. Avaliação espaço-temporal da relação entre o estado trófico do reservatório de Barra Bonita (SP) e o potencial poluidor de sua bacia hidrográfica. Sociedade \& Natureza, v. 19, n. 2, p. 5-18, 2007. http://dx.doi.org/10.1590/S1982-45132007000200001

RAJANKAR, P. N.; TAMBEKAR, D. H.; WATE, S. R. Groundwater quality and water quality index at Bhandara District. Environmental Monitoring Assessment, v. 179, p. 619-625, 2011. http://dx.doi.org/10.1007/s10661-010-1767-y

RÍOS-VILLAMIZAR, E. A.; MARTINS JUNIOR, A. F.; WAICHMAN, A. V. Caracterização físico-química das águas e desmatamento na bacia do rio Purus, Amazônia Brasileira Ocidental. Revista Geografia Acadêmica, v. 5, n. 2, 2011.

SILVA, G. S.; JARDIM, W. F. Um novo índice de qualidade das águas para proteção da vida aquática aplicado ao Rio Atibaia, região de Campinas/Paulínia - SP. Química Nova, v. 29, p. 689-694, 2006. http://dx.doi.org/10.1590/S0100-40422006000400012

SOUZA, A. P.; MOTA, L. L.; ZAMADEI, T.; MARTIN, C. C.; ALMEIDA, F. T.; PAULINO, J. Classificação climática e balanço hídrico climatológico no estado de Mato Grosso. Nativa - Pesquisas Agrárias e Ambientais, v. 1, n. 1, p. 34-43, 2013. http://dx.doi.org/10.14583/2318-7670.v01n01a07

TOLEDO, L. G.; NICOLELlA, G. Índice de qualidade de água em microbacia sob uso agrícola e urbano. Scientia Agrícola, v. 59, p. 181-186, 2002.

http://dx.doi.org/10.1590/S0103-90162002000100026 
TRINDADE, A. L. C. Aplicação de técnicas estatísticas para avaliação de dados de monitoramento de qualidade das águas superficiais da porção mineira da bacia do rio São Francisco. 2013. 165f. Dissertação (Mestrado em Saneamento, Meio Ambiente e Recursos Hídricos) - Universidade Federal de Minas Gerais, Belo Horizonte, 2013

VANZELA, L. S.; HERNANDEZ, F. B. T.; FRANCO, R. A. M. Influência do uso e ocupação do solo nos recursos hídricos do Córrego Três Barras, Marinópolis. Revista Brasileira de Engenharia Agrícola e Ambiental, v. 14, p. 55-64, 2010. http://dx.doi.org/10.1590/S1415-43662010000100008

WITTMAN, J.; WECKWERTH, A.; WEISS, C.; HEYER, S.; SEIBERT, J.; KUENNEN, B. et al. Evaluation of land use and water quality in an agricultural watershed in the USA indicates multiple sources of bacterial impairment. Environmental Monitoring Assessment, v. 185, p. 10395-10420, 2013. http://dx.doi.org/10.1007/s10661-013$3340-\mathrm{y}$

WU, J.; CHENG, S.; LI, Z.; GUO, W.; ZHONG, F.; YIN, D. Case study on rehabilitation of a polluted urban water body in Yangtze River Basin. Environmental Science and Pollution Research, v. 20, p.7038-7045, 2013. http://dx.doi.org/10.1007/s11356-012$1351-9$

ZANINI, H. L. H. T.; AMARAL, L. A.; ZANINI, J. R.; TAVARES, L. H. R. Caracterização da água da microbacia do córrego Rico avaliada pelo índice de qualidade de água e de estado trófico. Engenharia Agrícola, v. 30, p. 732-741, 2010. http://dx.doi.org/10.1590/S0100-69162010000400017

ZHANG, C.; APPEL, E.; QIAO, Q. Heavy metal pollution in farmland irrigated with river water near a steel plant - magnetic and geochemical signature. Geophysical Journal International, v. 192, p. 963-974, 2013. http://dx.doi.org/10.1093/gji/ggs079 